REVISTA 89.1

Revista RELACIONES INTERNACIONALES

Escuela de Relaciones Internacionales.

Universidad Nacional, Costa Rica.

N. ${ }^{\circ} 89.1$ • Enero-Junio de 2016

doi: http://dx.doi.org/10.15359/ri.89-1.5

Pp. 95-121

\title{
HOTEL FINCA CAMPESTRE ROSA BLANCA: EJEMPLO DE RESPONSABILIDAD SOCIAL EMPRESARIAL EN COSTA RICA
}

\author{
Kattia Lizzett Vasconcelos Vásquez* \\ Claudia Marín Gutiérre**
}

\section{RESUMEN:}

Este artículo trata la relación entre la Certificación de Sostenibilidad Turística (CST) en Costa Rica, el cual ha implantado un sistema de gestión basado en el estándar CST y la Responsabilidad Social Empresarial (RSE). La valoración permitirá encontrar diferencias o similitudes entre la CST y la RSE, con el fin de ver cómo estas favorecen la gestión empresarial y la sostenibilidad, poniendo de manifiesto los principales cambios organizativos que se deben implementar en la institución o empresa, para lograr un resultado eficaz y un mejor servicio al cliente.

Palabras clave: Responsabilidad social ambiental empresarial, turismo responsable, hotel responsable, Certificación de Sostenibilidad Turística, caso de estudio.

\begin{abstract}
:
The focus of this article is the relationship between the Certification for Sustainable Tourism (CST) in Costa Rica, which has implemented a management system based on the CST standard, and the Corporate Social Responsibility (RSE). The assessment will find differences and / or similarities between CST and CSR in order to see how they enhance business management and sustainability, and will also highlight the major organizational changes that must be implemented in the institution or company to achieve an effective outcome and better customer service.

Keywords: Environmental Social Corporate Responsibility; Responsible Tourism; Responsible Hotel; Certification of Sustainable Tourism; Case Study.
\end{abstract}

* Catedrática de la Universidad Nacional de Costa Rica, Académica de la Escuela de Administración. Estudiante del Doctorado de la Universidad de Valencia España en Estrategia y Calidad. Máster en Administración de Empresas con énfasis en Mercadeo. Licenciada en Administración énfasis Recursos Humanos. Correo electrónico: kvascon@una.cr

** Doctora en Educación con especialidad en Mediación Pedagógica. Máster en Ciencias de la Educación con Mención en Educación Preescolar. Licenciada en Ciencias de la Educación con Énfasis en Administración de Servicios Sociales Infantiles. Licenciada en Teatro con énfasis en Teatrología de la Universidad Nacional. Investigadora. En la actualidad, forma parte del equipo de trabajo de la academia de la Universidad Hispanoamericana en la Facultad de Educación. Correo electrónico: chammue14@yahoo.es 


\section{Introducción}

En la actualidad, las certificaciones adquieren una importancia destacada entre las organizaciones en la mayoría de los entornos en los que se desenvuelven. La consecución de una certificación permite demostrar a terceros, ya sean estos usuarios, clientes individuales o empresariales, que se cumple con los requisitos técnicos definidos en una norma previamente establecida por algún organismo de reconocido prestigio. Si bien, existen diversidad de normas, las más reconocidas por organismos nacionales e internacionales encargados de vigilar por la excelencia y la calidad de los servicios y productos son las procedentes de la Organización Internacional de Estandarización (International Organization for Standardization, abreviado como ISO).

A nivel mundial existen cientos de investigaciones sobre empresas certificadas, como por ejemplo: Esfuerzo y Eficacia en los Sistemas de Gestión Medioambiental de Empresas Certificadas ISO 14001 (Salamanca, España), Instituto Politécnico Nacional: Plan estratégico para el mantenimiento y mejora del sistema de gestión de la calidad de empresas del sector industrial certificadas en ISO 9001 (México), Empresas excelentes entre las certificadas en las normas ISO 9000 (Valencia, España), Estadísticas de empresas certificadas en ISO y muchas otras.

Este artículo se centrará en la Certificación de Sostenibilidad Turística, en adelante CST, ya que esta es producto de una estrategia nacional como parte de marca país. Esta última entendida como una etiqueta de calidad y confiabilidad en los productos y servicios que genera el país en las diferentes áreas empresariales, no solamente en el área del turismo, sino también en el área, por ejemplo, de la producción de llantas, donde puede mencionarse la empresa Bridgestone Latinoamérica Norte.

La importancia de lo anterior para el país es que esta etiqueta genera confiabilidad y prestigio en los productos y servicios que se producen y se ofertan, forjando, así, mejores ingresos para el país y reconocimiento nacional e internacional.

Por este motivo se consideró de gran impacto social, dar a conocer el caso del Hotel Finca Campestre Rosa Blanca, como un ejemplo de Responsabilidad social empresarial en Costa Rica, por medio del presente artículo.

Con respecto al área del turismo, según los datos que presenta la Asociación Empresarial de Desarrollo de la Cámara de Industria de Costa Rica, el movimiento de turismo responsable ha crecido vertiginosamente. Esto también es apoyado por 
Riotte (2015), que cuenta con más de 35 años de experiencia en el área de turismo en Costa Rica y es codueña de la empresa: Agencia de Viajes Brazos Abiertos (CANATUR), dedicada al turismo responsable, quien señala que: “... la solicitud a las empresas denominadas 'DMC' (empresas organizadoras de eventos empresariales con responsabilidad social) crece cada día más, los requisitos de los eventos deben llevar sello verde con proyección a la naturaleza" (párr. 2).

\section{Costa Rica: Un destino ecoturístico}

El ecoturismo es aquel segmento especializado del turismo responsable, que promueve y apoya la conservación de la naturaleza y los valores culturales de los destinos, los interpreta para el cliente, favorece la mejora socioeconómica de las comunidades locales y persigue sensibilizar y satisfacer, de manera ética, a su clientela. Es decir, diseña las actividades en consonancia con el ambiente que le rodea y pone a sus clientes (turistas) en contacto directo y personal con la naturaleza y la cultura local.

Como menciona Flores (2014, p. 36):

Costa Rica cuenta con un posicionamiento a nivel internacional como el primer destino líder en ecoturismo y tiene una larga trayectoria en turismo sostenible, un concepto que logra un equilibrio entre su desarrollo económico, respetando el patrimonio e incluyendo a las comunidades como parte de ese crecimiento, lo que produce que cientos de turistas valoren a nuestro país como un destino por excelencia y responsable.

Aunado a lo que menciona Flores (2014), el Instituto Costarricense de Turismo (ICT) (2014), en el Informe de avance sobre el Plan Nacional de Turismo 20102016, reafirma que Costa Rica tiene una imagen de país de turismo responsable, respaldado por la amplia riqueza natural; aunque solamente posea un $0,03 \%$ de espacio del planeta, en sus océanos se encuentran 6777 especies, que equivalen al 3,5\% de especies conocidas en la Tierra.

Autores como Napier (1997) y Lindberg y Aylward (1999), manifiestan que al ser Costa Rica el precursor en este tipo de turismo, se convierte en uno de los principales destinos "verdes" del mundo. Por ejemplo, para Napier (1997), "Costa Rica tiene la reputación del paraíso ecológico" (p. 18), y agrega que: "Este país, muestra la relación entre naturaleza, belleza natural y la combinación de parques nacionales especializados" (p. 63). También Lindberg y Aylward (1999) enriquecen esta visión cuando expresan: "Costa Rica es uno de los lugares en el mundo con mayor biodiversidad, además es un país con una 
experiencia a nivel internacional sobre ecoturismo y manejo de parques nacionales especializados" (p. 56).

Como puede observarse con las citas anteriores, Costa Rica no solo tiene una fuerte reputación de "paraíso ecológico", sino también se le reconoce la biodiversidad y la experiencia internacional, tanto en ecoturismo, como en manejo de parques nacionales, lo que lo proyecta con fuerza fuera de sus fronteras, con el impacto que esto tiene a nivel de divisas, tan importantes para su desarrollo.

De acuerdo con el Instituto Costarricense de Turismo (2015), en este año, la cantidad de divisas por turismo fue de $\$ 2.253,30$ millones, superando la exportación de café, banano y piña; con una consulta consecutiva a la información en la misma institución se encontró que para el año 2014 la cifra incrementó en \$ 2.636 millones y para el 2015, según Barquero (2016), en un artículo del periódico La Nación, estas divisas aumentaron aún más, pasando de $\$ 2.253,30$ millones en 2013 a \$ 2.882 millones en 2015.

De acuerdo con lo que plantea Barquero (2016), en su artículo y la información encontrada en el Instituto Costarricense de Turismo (ICT), es muy importante resaltar que se observa un crecimiento en la actividad turística del 5,5\% en los últimos dos años, lo que corresponde a 2.665 .000 visitantes al país, lo que se refleja en el ingreso de las divisas en un $9 \%$.

Se hace necesario, entonces, destacar que el buen manejo de los recursos turísticos, que se lleva a cabo en la actualidad en Costa Rica, ha permitido que los ingresos por este concepto hayan subido en un $8 \%$.

$\mathrm{Al}$ respecto, Flores (2014), señala que:

Costa Rica tiene una marca muy bien posicionada en materia de turismo sostenible. Para nadie es un secreto que muchas empresas quieren venir al país a organizar sus reuniones y vivir la experiencia de mezclar su visita con el encanto que ofrecen otros de nuestros atractivos. (p. 23)

Según la información que se desprende de Flores (2014) y de Barquero (2016), se puede concluir que, para Costa Rica, el turismo se ha convertido en el motor de la economía nacional, pues su actividad ha crecido tanto, que superó el ingreso de sus divisas tres veces el crecimiento de la economía en general.

Cabe mencionar también que el mismo Flores (2014), complementa lo anterior con lo siguiente: 
El turismo en Costa Rica representa el 4,9\% del Producto Interno Bruto y genera cerca de 100,000 empleos en forma directa y de 250,000 en forma indirecta lo que representa el $12 \%$ del empleo total del país... Cerca del $58 \%$ de los turistas internacionales que visitan Parques Nacionales generan cerca de US \$ 8 millones de ingresos por entradas a dichos parques. Estos ingresos favorecen la conservación de áreas protegidas y también se emplean para la educación ambiental y concientización sobre la conservación en función de una nueva cultura turística. (p. 4-5)

De acuerdo con lo que exponen algunos especialistas en el sector turístico, a nivel nacional e internacional, como Suyen (2013), Domínguez (2012), el Instituto Costarricense de Turismo (2014) y otros, es importante el desarrollo turístico con responsabilidad social, por el impacto positivo que puede traer tanto individual como colectivamente; por ejemplo, la generación de mayores oportunidades de empleo, el desarrollo de las comunidades por el ingreso de dinero, venta de artículos propios de la zona y sus productos agrícolas, y otros. Todo ello puede dar a conocer la región a nivel nacional e internacional, beneficia directamente a las familias y logra un desarrollo en las comunidades.

Por el contrario, el impacto negativo puede darse tanto a nivel de salud, economía, ambiente, cuando se contaminan ríos, se cierran fuentes de empleo, se concentran focos de contaminación en comunidades, lo que puede acarrear problemas de salud en las personas y convierte a las comunidades que la padecen, en vulnerables; de igual forma se puede mencionar la afectación negativa en los ecosistemas, al punto de hacerlos desaparecer, entre muchos otros problemas.

Bien (2008), también menciona que: “Costa Rica, como líder del turismo sostenible, ha sabido proyectarse exitosamente en este campo. Para ello ha contado con el gobierno que diseña e impulsa la ejecución de las políticas y acuerdos internacionales sobre desarrollo sostenible" (p. 8). En este sentido, el Instituto Costarricense de Turismo (ICT) propuso el programa de Certificación para la Sostenibilidad Turística, conocido por las siglas "CST".

\section{La Certificación de Sostenibilidad Turística (CST)}

En la actualidad, la CST está teniendo un gran desarrollo porque, por un lado, los resultados se pueden observar a corto plazo y, por el otro, el Banco Interamericano de Desarrollo (BID) ha pedido, como requisito esta certificación para apoyar nuevos proyectos turísticos en el área centroamericana, de tal forma que, las empresas interesadas tienen que someterse a este proceso de evaluación y, en 
particular, lo que corresponde al apartado de sostenibilidad de proyectos turísticos, cuya referencia es la CST de Costa Rica.

Es importante destacar que el BID toma como referencia la CST de Costa Rica por el impacto positivo que ella tiene, como se mencionó anteriormente; pero en este documento no se desarrollan los temas del origen, implementación, ventajas y desventajas, porque ello responde a otro estudio de caso.

Sí es importante indicar que, dentro de las metas del Instituto Costarricense de Turismo (ICT) (2014), para el año 2016 se espera contar con 500 empresas con CST (en la actualidad hay 320 empresas certificadas), además de: “... ser el primer destino líder ecoturístico con una larga trayectoria en turismo sostenible” (p. 10).

La misma institución (2014) menciona que otra de las metas es aumentar, en un $5 \%$, la cantidad de turistas (2.700.000 turistas internacionales), que Costa Rica se convierta en un destino: "Clásico de naturaleza" con las estrategias que ha implementado el ICT:

- Diferenciación de los destinos actuales y nuevos.

- Mejoramientos en las capacidades.

- Fortaleciendo el perfil deseado". (p. 20)

Pero es importante destacar, en este punto que, si bien es cierto el ICT tiene como meta la certificación de 500 empresas para el año 2016, también es cierto que le interesa más la calidad de turistas que la cantidad de los visitantes, por lo que pretende fortalecer el programa de Certificación de Sostenibilidad Turística, cuyo fortalecimiento lo ha tenido como meta permanente.

No obstante lo anterior, también hay que anotar con respecto a la norma, que aunque ha tenido favorables resultados, al igual que cualquier regla o política, cuenta con aspectos positivos y negativos que afectan diversos ámbitos, como por ejemplo, el institucional, el profesional, el de servicio al cliente y otros.

De la misma manera, hay que resaltar que el esfuerzo conjunto entre diversos organismos capitaneados por el ICT -que se ocupa de regular y proteger el campo turístico, comprometiéndose a resguardar tanto la preservación en el tiempo, como la mejora constante en los productos sostenibles de alta calidad que se ofrecen- han llevado al turismo en Costa Rica a impactar exitosamente el mercado y a tener el reconocimiento internacional con el que cuenta hoy. 
Para apoyar lo anterior, Flores (2014), que menciona:

Este trabajo conjunto se ve plasmado en el Certificado para la Sostenibilidad Turística (CST). Es así como las empresas turísticas costarricenses que voluntariamente han decidido concretar su responsabilidad con el medio ambiente, garantizan una experiencia para el turista que aparte de ser sostenible con los recursos naturales; ofrece la mayor calidad en sus servicios y gran compromiso por medio de sus programas de responsabilidad social. (p. 36)

Paralelo a la CST, en el país también se está gestando la estrategia de la Responsabilidad Social Empresarial, entendida de ahora en adelante como (RSE), que permitirá mejorar, aún más, la calidad y compromiso de estas y las futuras empresas.

En este punto preciso, se considera necesario aclarar la relación que existe entre CST y RSE, donde la primera se entiende como: una etiqueta de calidad y confiabilidad en los productos y servicios que genera el país en las diferentes áreas empresariales, como se menciona en el presente apartado; y la segunda, se entiende como: aquella responsabilidad que tienen las organizaciones ante los impactos que provocan sus decisiones y actividades, tanto en la sociedad como en el medio ambiente, tal como se desarrollará en el siguiente apartado.

\section{La responsabilidad social empresarial}

El Estado costarricense se ha pronunciado y ha emitido el proyecto de ley 17510, que establece la generación de una certificación denominada "Socialmente Responsable" que formaría parte del programa denominado "Costa Rica Califica”; aunque aún no es ley, varias empresas se han acogido en forma voluntaria a este programa, según la propuesta de ley Marco de la RSE en el año 2009.

De igual manera, la responsabilidad social tiene varios enfoques, para el caso de Costa Rica, se ha dado la siguiente definición de la RSE facilitada por ALIARSE et al. (2009):

... la responsabilidad de una organización ante los impactos de sus decisiones y actividades en la sociedad y el medio ambiente, a través de un comportamiento transparente y ético que contribuya al desarrollo sostenible, la salud y al bienestar general de la sociedad tomando en consideración las expectativas de sus partes interesadas; cumplir con la legislación aplicable y sea consistente con la normativa internacional de comportamiento; y está integrada en toda la organización y se lleve a la práctica en sus relaciones. (p. 9) 
En ese sentido, es importante recalcar aspectos destacados como sostenibilidad, la legislación, el ambiente y la sociedad. Se cree importante indicar que el desarrollo de la RSE en Latinoamérica ha sido diferente de otros lugares y que se reconoce que hay aportes desde los diferentes países; por ejemplo, el GAB (good agricultural practices) implementado por Chile y, en el Caso de Costa Rica, el aporte de mayor reconocimiento es la CST (Reficco y Ogliastri, 2009), que fue desarrollado en el apartado anterior.

Según la Asociación Empresarial para el Desarrollo, de ahora en adelante AED, Costa Rica ha adoptado una definición de RSE de tercera generación. De manera concreta y para AED (2004), se ha conceptualizado la RSE como: “... el compromiso permanente de las empresas para aumentar su competitividad mientras contribuyen activamente al desarrollo sostenible de la sociedad costarricense mediante acciones concretas y medibles dirigidas a solucionar los problemas prioritarios del país" (p. 32).

Para ampliar qué es la III Generación de la RSE, hay que aclarar que esta se denomina como: Competitividad Responsable y, de acuerdo con lo que argumenta AED (2014), hay una serie de esfuerzos individuales y grupales a nivel del Estado costarricense, que apoyan el realizado por las empresas involucradas y que, desde el año 2014, ha venido implementando una estrategia llamada Costa Rica inclusiva, medida que integra, en el mercado laboral, a personas que presentan discapacidades especiales.

Esta estrategia es llamada por Grant (2010), como Nuevo Milenio y lo interesante de ella es que, tanto la RSE como la CST, pueden complementarse mutuamente porque, para el sector hotelero en el caso de Costa Rica, ambas son similares.

Al respecto Domínguez (2012), Porter y Kramer (2006) hacen referencia a que esta es una estrategia empresarial que se puede situar, tanto en el nivel de los negocios como en el de las corporaciones. Al convertirse la RSE en una estrategia empresarial, como tal, no se observa o advierte diferencia del negocio en el día con día, debido a que está inmersa en los planes y acciones que la empresa desarrollo diariamente.

Lo señalado anteriormente responde, en el caso del turismo en Costa Rica, a los diferentes esfuerzos que realizan las empresas que en este momento se encuentran trabajando con la CST y RSE, realizando proyectos en diferentes ejes de RSE y que aportan tanto en el área educativa y ambiental como cultural. Algunas de estas empresas son: trabajo conjunto del proyecto Creciendo Juntos Libros para Todos: Ministerio de Educación, Instituto Costarricense de Electricidad, 
Universidad de Costa Rica, Instituto de Acueductos y Alcantarillados, Hotel Punta Islita, empresa Ríos Tropicales y muchas otras. Seguidamente se hace referencia al trabajo realizado por algunas empresas en el eje comunidad y en el eje ambiental.

\section{Eje comunidad:}

Creciendo Juntos Libros para Todos ha estado funcionando en los últimos 15 años, trabajando con la comunidad donde 21 centros educativos han sido beneficiados, incluyendo aproximadamente 4.428 personas. El caso del proyecto de creciendo juntos inició en el año 2000, con un diagnóstico de necesidades de la zona de Guanacaste, por parte de un grupo de empresas (que se unieron para mejorar las condiciones de las comunidades) donde la educación y capacitación resultaron las mayores necesidades detectadas. El eje en este caso es hacia la comunidad y participan el grupo empresarial junto con el Ministerio de Educación, el Instituto Costarricense de Electricidad, la Universidad de Costa Rica y el Instituto de Acueductos y Alcantarillados, cada organización desde su posibilidad.

Hotel Punta Islita. Museo de Arte Contemporáneo con más de 12 años. Este hotel realizó, en el año 2002, el eje hacia la comunidad con un museo al aire libre, único en Latinoamérica, que para muchas personas se ha convertido en el proyecto de vida, este favorece a miembros de la comunidad y de otras comunidades vecinas. Un ejemplo de gran valor de los productos generados es entregado en la Cumbre de La Comunidad de Estados Latinoamericanos y Caribeños, realizado en Costa Rica en el año 2015: “... el recuerdo que se les brindó a los participantes de la CELAC, Costa Rica en el mes de enero de año 2015, que fue una cantidad de 2500 de ellos" (p. 15).

\section{Eje Ambiente:}

La empresa Ríos Tropicales ha sembrado mil millones de árboles en los últimos 26 años, trabajando en actividades de rafting, con varios campeonatos mundiales organizados.

De igual manera, el Programa de Educación Ambiental en Escuelas durante 26 años, trabajando en actividades de educación ambiental en la zona.

Además, de acuerdo con AED (2013), el Proyecto Áreas Protegidas de la Agencia de viajes Horizontes. La era de la sostenibilidad, en 31 años de ecoturismo y 7 años con la fundación. 
De igual manera, Reficco y Ogliastri (2009) reconocen la importancia de la Certificación para la Sostenibilidad Turística, implementada por el Instituto Costarricense de Turismo en el año 1997 y por la Comisión Nacional de Acreditación, que fue establecida oficialmente desde el año 1997 y que está “... orientada a la competitividad, la sostenibilidad ambiental y el desarrollo de sus grupos involucrados, o grupos de interés" (p. 6).

Por lo indicado en los párrafos anteriores, surge la inquietud de establecer la relación entre la CST y la RSE. De esta manera, se estaría contribuyendo a determinar si es viable esta correlación. Se toma como caso el Hotel Finca Campestre Rosa Blanca por ser pionero en la adquisición de la CST, además, por ostentar la máxima puntuación en la certificación y por la disposición hacia las nuevas alternativas de gestión administrativas, pues se encuentran anuentes a realizar implementaciones de esta estrategia en ese sentido.

\section{Perfil del caso}

A continuación se presentan los aspectos generales del hotel caso de estudio en CST y RSE.

Tabla 1.

Aspectos generales del Hotel Finca Campestre Rosa Blanca

\begin{tabular}{|l|l|}
\hline \multicolumn{1}{|c|}{ Datos } & \multicolumn{1}{c|}{ Detalles } \\
\hline Dirección & $\begin{array}{l}800 \text { norte del centro de distribución de } \\
\text { Café Britt, Santa Bárbara, Heredia }\end{array}$ \\
\hline Provincia & Heredia \\
\hline Cantón & Santa Bárbara \\
\hline Tipo de hotel & Montaña \\
\hline Gerente & Johnny Alvarado \\
\hline Teléfono(s) & $(506) 22699392,(506) 22693333$ \\
\hline Fax & $(506) 22699555$ \\
\hline Habitaciones & 9 \\
\hline Estrellas & 4 \\
\hline Fecha de certificación & $17-11-2014$ \\
\hline Nivel & 5 \\
\hline Evaluador & EQUIPO TECNICO ICT \\
\hline
\end{tabular}




\begin{tabular}{|l|l|}
\hline \multicolumn{1}{|c|}{ Datos } & \multicolumn{1}{c|}{ Detalles } \\
\hline Correo electrónico & $\begin{array}{l}\text { dsoto@fincarosablanca.com; rblanca@ } \\
\text { racsa.co.cr; info@fincarosablanca.com }\end{array}$ \\
\hline Pagina web & www.finca-rblanca.co.cr \\
\hline
\end{tabular}

Nota: CST (2015).

Los datos presentados en la tabla 1 señalan que la acreditación más reciente se dio en el mes de noviembre del año anterior y los resultados obtenidos por cada ámbito se muestran en la tabla 2:

\section{Tabla 2.}

Resultados de la evaluación de la CST aplicado al Hotel Finca Campestre Rosa Blanca

\begin{tabular}{|l|c|}
\hline NIVEL & $\mathbf{5}$ \\
\hline ÁMBITO & Puntuación porcentual \\
\hline Planta de servicio & 100.00 \\
\hline Cliente & 100.00 \\
\hline Entorno socioeconómico & 101.00 \\
\hline Entorno físico biológico & 104.00 \\
\hline
\end{tabular}

\section{Nota: CST (2015).}

De la tabla 2 se obtiene que en el ámbito de planta de servicio el hotel logra un $100.00 \%$. En la inspección realizada en las instalaciones se pudo apreciar el aprovechamiento de los recursos naturales en pro de la gestión operativa, los paneles solares, las zonas de aprovechamiento del sol para secar toallas, sabanas, mantelería y otros de manera que la generación de energías alternativas están presentes.

En el ámbito de cliente, el Hotel Finca Campestre Rosa Blanca obtiene un $100.00 \%$ donde, verificando con el cuestionario esta situación, no se presentan carencias o debilidades sino, más bien, una serie de estrategias implementadas en favor de todos los involucrados, lo que hace posible que el Hotel Finca Campestre Rosa Blanca supere el estándar dado en la CST.

Lo anterior se puede apreciar en ejemplos como: Capacitación y educación constante a los clientes internos y externos (huéspedes) en aspectos de protección de la 
naturaleza y del ambiente, uso y reutilización de los recursos materiales, junto a las estrategias de reciclaje y producción orgánica para el restaurante.

De igual manera, el ámbito socioeconómico obtiene un 101,00\%, lo que lo convierte en un hotel con unas características muy interesantes, como por ejemplo: ha asumido los productos de artesanía elaborados por personas adultas mayores del hogar de ancianos de Santa Bárbara, para que sean vendidos en la tienda del hotel y los recursos, producto de esas ventas, se inyectan directamente al Hogar. De igual manera, los trabajadores del hotel participan con sus comunidades dando capacitación a los vecinos para mejorar. También, los grupos culturales de la comunidad, propiamente la escuela del lugar, tiene participación directa en eventos de promoción cultural como bailes, danzas, teatro y otras actividades que promocionan la zona.

En el ámbito físico biológico donde el rubro fue $104,00 \%$, sobrepasando el 100.00 , nuevamente se ve la participación de la administración y todo el equipo que labora en el lugar, colaborando a lo interno y externo de las instalaciones, por ejemplo, la fumigación no solo se hace en el hotel sino con los vecinos. El personal del hotel tiene la actitud y la aptitud para denunciar situaciones que afecten el medio ambiente y esto se logra a través del empoderamiento que, según Margarita González', encargada de recursos humanos (Comunicación personal, 2014), les da la Gerencia del Hotel por medio de capacitaciones semanales que se brindan en el lugar, como por ejemplo: Cuido del medio ambiente, eliminación de focos de contaminación, manejo de desechos, deforestación y reforestación, cuido de áreas protegidas, otros.

Este hotel no solo supera la escala de la CST, sino que logra la máxima puntuación en el nivel de las 5 hojas y está en una constante mejora dentro y fuera de la empresa. Según lo que menciona la encargada de RRHH (Comunicación personal, 2014), lo anterior lo logra mediante un grupo de trabajadores denominados clientes internos, quienes son trabajadores del hotel en cuyas labores toman un rol de clientes, accediendo a todos los servicios que brinda el hotel y evaluándolos de forma constante y rigurosa, apuntando siempre a conseguir la excelencia, de forma tal que, si alguno de los servicios no funciona o no está acorde con lo que plantean las normas en esa meta de excelencia, rápidamente es corregido.

1 Entrevista a profundidad sobre CST y RSE en el Hotel Finca Campestre Rosa Blanca, Santa Bárbara de Heredia, Costa Rica. 
Tabla 3.

Aspectos generales del cliente interno del Hotel Finca Campestre Rosa Blanca

\begin{tabular}{|c|c|c|}
\hline ASPECTO & RANGO & NÚMERO \\
\hline \multirow{2}{*}{ DISTRIBUCIÓN POR SEXO } & MASCULINO & 20 \\
\hline & FEMENINO & 12 \\
\hline COLOR DE PIEL ${ }^{2}$ & BLANCOS & 32 \\
\hline \multirow{5}{*}{ EDAD } & MENOR DE 18 AÑOS & 00 \\
\hline & MENOR DE 28 AÑOS & 07 \\
\hline & MENOR DE 38 AÑOS & 21 \\
\hline & MENOR DE 48 AÑOS & 03 \\
\hline & MENOR DE 58 AÑOS & 01 \\
\hline \multirow{3}{*}{ ESTADO CIVIL } & SOLTEROS & 04 \\
\hline & DIVORCIADOS & 02 \\
\hline & UNIÓN LIBRE & 03 \\
\hline CANTIDAD DE PERSONAS & TOTAL & 32 \\
\hline
\end{tabular}

Nota: Entrevista a profundidad, 2014, encargada de Recursos Humanos del hotel.

En la tabla 3 se presentaron los principales datos correspondientes al personal del hotel, donde se muestra personal de ambos sexos y con variedad de edades. También, es importante destacar que el 100\% del personal es de la zona.

Este hotel tiene una política sobre capacitación constante en todas las áreas, desde las administrativas, con manejo de personal, de espacios y otros; en eje ambiental, por ejemplo, cuidado del ambiente, cursos de contaminación, reutilización, otros, y servicio al cliente (huésped), atención al cliente y otros.

2 En esta opción se incluían todos los tonos de piel. Sin embargo acá el resultado es único. 
Además, un aspecto muy importante de destacar es que todos los empleados conocen de todos los trabajos, no se especializan en uno solo, por ejemplo, el hotel no utiliza gafetes con el nombre de la persona y el puesto, solamente usa unos pedazos de metal en forma rectangular y de dos dedos de ancho y 5 centímetros de largo, estéticos, conocidos como placas, donde se encuentra escrito el nombre de cada quien de los miembros del personal; estos se llevan colocados en el lado derecho superior del pecho.

El hecho de que en estos objetos llamados placas solo aparezca el nombre de la persona, y no nombre y puesto, busca que el empleado pueda realizar cualquier tipo de función, parte de lo que se desarrolla en las capacitaciones como política del hotel. Esta constituye una estrategia de empoderamiento y los faculta para hacer otras funciones.

Un detalle a rescatar dentro de las políticas y normas que se desarrollan en el lugar es que se busca, con ellas, no solo el bienestar del negocio, sino también el bienestar y la armonía de los empleados dentro del hotel. Por ejemplo, hay un funcionario que se desplaza a caballo desde su casa al trabajo y le fue asignada, por comodidad, una parte de la bodega (antiguo establo) para que este el caballo, su medio de transporte, como si fuera el automóvil o la bicicleta de los otros empleados, quienes también cuentan con un espacio para ello diferente, por supuesto, al del establo. Esta acción se convirtió después en un atractivo más para los visitantes.

Con respecto a la RSE, seguidamente, en la tabla 4, se presenta la relación que se da con parte de un grupo de ejes o áreas y cómo se reflejan estos en la realidad integral del Hotel Finca Campestre Rosa Blanca. 
Tabla 4.

Ejes o áreas, subejes y acciones de la RSE

en el Hotel Finca Campestre Rosa Blanca. Gobernabilidad

\begin{tabular}{|c|c|c|}
\hline EJE O ÁREA & SUB EJE & ASPECTO \\
\hline \multirow{7}{*}{ GOBERNABILIDAD } & Legalidad & $\begin{array}{l}\text { Es una empresa inscrita en el registro co- } \\
\text { rrespondiente. Cumple con lo establecido } \\
\text { en la normativa del país. }\end{array}$ \\
\hline & Principios éticos & $\begin{array}{l}\text { El hotel tiene y respeta un código de ética } \\
\text { en beneficio de la moral y la seguridad. }\end{array}$ \\
\hline & $\begin{array}{l}\text { Cultura organiza- } \\
\text { cional }\end{array}$ & Se maneja un ambiente familiar. \\
\hline & $\begin{array}{l}\text { Gobernabilidad } \\
\text { corporativa }\end{array}$ & $\begin{array}{l}\text { Es algo que se está implementando como } \\
\text { país. En este caso el Hotel tiene una alianza } \\
\text { estratégica con los diferentes públicos. }\end{array}$ \\
\hline & $\begin{array}{l}\text { Transparencia } \\
\text { con la comunidad }\end{array}$ & $\begin{array}{l}\text { Hay una relación comercial con algunas } \\
\text { organizaciones de la comunidad. }\end{array}$ \\
\hline & $\begin{array}{l}\text { Propiedad } \\
\text { intelectual }\end{array}$ & $\begin{array}{l}\text { No aparece patentado nada en el registro } \\
\text { público. }\end{array}$ \\
\hline & $\begin{array}{l}\text { Gestión del } \\
\text { riesgo }\end{array}$ & $\begin{array}{l}\text { Es algo que apenas el país comienza a de- } \\
\text { sarrollar (Este eje se desarrolla en el ámbi- } \\
\text { to financiero bancario). }\end{array}$ \\
\hline
\end{tabular}

Nota: Instrumentos aplicados (2014).

Cumplir con lo establecido en el marco jurídico del país, la relación con la comunidad y la transparencia con que su gestión se realiza son muestra de que se está llevando a cabo la RSE, aspecto que se encuentra en ambas estrategias de gestión organizacional. 
Tabla 5 .

Ejes o áreas, sub ejes y acciones de la RSE

en el Hotel Finca Campestre Rosa Blanca. Público interno y mercadeo

\begin{tabular}{|c|c|c|}
\hline EJE O ÁREA & SUB EJE & ASPECTO \\
\hline \multirow{5}{*}{$\begin{array}{l}\text { PÚBLICO } \\
\text { INTERNO }\end{array}$} & $\begin{array}{l}\text { Condiciones } \\
\text { de trabajo }\end{array}$ & $\begin{array}{l}\text { La empresa utiliza personas del } \\
\text { ámbito comunal o nacional para } \\
\text { trabajar en el nivel administrati- } \\
\text { vo o gerencial. }\end{array}$ \\
\hline & $\begin{array}{l}\text { Diálogo y } \\
\text { participación }\end{array}$ & $\begin{array}{l}\text { Los empleados del hotel conocen } \\
\text { los alcances de la misión y las } \\
\text { políticas para la sostenibilidad. }\end{array}$ \\
\hline & $\begin{array}{l}\text { Respeto al } \\
\text { individuo }\end{array}$ & $\begin{array}{l}\text { La empresa establece acciones } \\
\text { prácticas que desincentivan el } \\
\text { acoso sexual y promueven la } \\
\text { igualdad de oportunidades para } \\
\text { ambos sexos. }\end{array}$ \\
\hline & $\begin{array}{l}\text { Desarrollo del } \\
\text { colaborador }\end{array}$ & $\begin{array}{l}\text { Techo corto por el tamaño de la } \\
\text { empresa. }\end{array}$ \\
\hline & $\begin{array}{l}\text { Promoción } \\
\text { del uso de la } \\
\text { mano de obra } \\
\text { local }\end{array}$ & Se contrata personal de la zona. \\
\hline
\end{tabular}




\begin{tabular}{|c|c|c|}
\hline EJE O ÁREA & SUB EJE & ASPECTO \\
\hline \multirow{3}{*}{ MERCADEO } & $\begin{array}{l}\text { Política de } \\
\text { mercadeo y } \\
\text { comunicación }\end{array}$ & $\begin{array}{l}\text { En el sitio web indican varias de } \\
\text { sus políticas. } \\
\text { La empresa declara bajo jura- } \\
\text { mento que toda la información } \\
\text { contenida en su material promo- } \\
\text { cional es estrictamente veraz. } \\
\text { La empresa tiene programas } \\
\text { específicos que promueven el } \\
\text { turismo nacional en los que se } \\
\text { ofrecen facilidades y tarifas } \\
\text { reducidas. } \\
\text { El hotel prohíbe el uso y promo- } \\
\text { ción de actividades de comercio } \\
\text { sexual, prostitución, expendio } \\
\text { de drogas u otros problemas } \\
\text { sociales. }\end{array}$ \\
\hline & $\begin{array}{l}\text { Excelencia en } \\
\text { la atención al } \\
\text { cliente }\end{array}$ & $\begin{array}{l}\text { La habitación cuenta con la } \\
\text { información y facilidades nece- } \\
\text { sarias para que el cliente pueda } \\
\text { hacer separación de las basuras. }\end{array}$ \\
\hline & $\begin{array}{l}\text { Calidad y } \\
\text { seguridad en } \\
\text { los servicios y } \\
\text { productos }\end{array}$ & $\begin{array}{l}\text { La empresa cuenta con un análi- } \\
\text { sis actualizado bimensualmente } \\
\text { de calidad de agua y hielo para } \\
\text { consumo humano extendido por } \\
\text { un ente autorizado. } \\
\text { De preferencia, el hotel está } \\
\text { utilizando productos frescos para } \\
\text { preparar y servir alimentos. }\end{array}$ \\
\hline
\end{tabular}

Nota: Instrumentos aplicados (2014).

En la tabla 5 anterior se presentan resultados de cómo se pone en práctica la RSE por parte del hotel. Un ejemplo de esta RSE aplicada es el personal es de la localidad, la utilización de recursos de la zona y el respeto por la cultura de la zona. 
Lo anterior se refleja en los buenos resultados que obtiene el hotel, la estrategia de mercadeo es social y con proyectos de los mismos empleados y de la comunidad en general. Eso le ha otorgado premios y reconocimientos por parte de viajeros, entre algunos de ellos, estadounidenses, alemanes, españoles, otros.

\section{Tabla 6.}

Ejes o áreas, subejes y acciones de la RSE

en el Hotel Finca Campestre Rosa Blanca. Proveedores y medio ambiente

\begin{tabular}{|l|l|l|}
\hline \multicolumn{1}{|c|}{ EJE O ÁREA } & \multicolumn{1}{|c|}{ SUB EJE } & \multicolumn{1}{c|}{ ASPECTO } \\
\hline \multirow{2}{*}{ PROVEEDORES } & $\begin{array}{l}\text { Selección } \\
\text { de proveedores }\end{array}$ & $\begin{array}{l}\text { Los productos en conserva necesarios se } \\
\text { compran en envases grandes y se prefie- } \\
\text { ren los envases de vidrio o los de metal } \\
\text { reciclable. }\end{array}$ \\
\cline { 2 - 3 } & $\begin{array}{l}\text { Relación } \\
\text { de proveedores }\end{array}$ & $\begin{array}{l}\text { La empresa tiene una relación comercial } \\
\text { o de apoyo permanente con al menos } \\
\text { una microempresa regional o nacional, } \\
\text { de carácter turístico o no, con la cual } \\
\text { colabora. }\end{array}$ \\
\hline \multirow{2}{*}{ MEDIO AMBIENTE } & $\begin{array}{l}\text { El hotel tiene su propia área de reserva } \\
\text { natural. }\end{array}$ \\
\hline & $\begin{array}{l}\text { La empresa está implementando una } \\
\text { campaña para el ahorro de agua, dirigi- } \\
\text { da a huéspedes y empleados. }\end{array}$ \\
\hline $\begin{array}{l}\text { Administración } \\
\text { insumos de la } \\
\text { empresa }\end{array}$ & $\begin{array}{l}\text { La empresa está utilizando fuentes de } \\
\text { energía alternativa para el calentamien- } \\
\text { to o precalentamiento de agua, bombeo } \\
\text { u otras. }\end{array}$ \\
\hline & $\begin{array}{l}\text { La empresa utiliza la ventilación natu- } \\
\text { ral, reflexión de luz y calor, sombra na- } \\
\text { tural y otros para procurar un ambiente } \\
\text { agradable. }\end{array}$ \\
\hline $\begin{array}{l}\text { La empresa mantiene un estricto control } \\
\text { periódico, en forma de registro, sobre } \\
\text { la composición y calidad de sus aguas } \\
\text { residuales. }\end{array}$ \\
\hline
\end{tabular}




\begin{tabular}{|c|c|c|}
\hline \multirow{13}{*}{ MEDIO AMBIENTE } & \multirow{13}{*}{$\begin{array}{l}\text { Administración } \\
\text { sostenible de } \\
\text { insumos de la } \\
\text { empresa }\end{array}$} & $\begin{array}{l}\text { Se han rotulado zonas contaminadas } \\
\text { para advertir sobre riesgos o peligros. }\end{array}$ \\
\hline & & $\begin{array}{l}\text { Las principales especies arbóreas están } \\
\text { debidamente identificadas con su nom- } \\
\text { bre local y nombre científico. }\end{array}$ \\
\hline & & $\begin{array}{l}\text { No se mantienen especies animales sil- } \\
\text { vestres en cautiverio. }\end{array}$ \\
\hline & & $\begin{array}{l}\text { El consumo de energía es monitoreado } \\
\text { continuamente por medio de medidores. }\end{array}$ \\
\hline & & $\begin{array}{l}\text { La empresa está utilizando al máximo la } \\
\text { iluminación natural. }\end{array}$ \\
\hline & & $\begin{array}{l}\text { Existe un programa de mantenimiento } \\
\text { preventivo para todos los equipos e ins- } \\
\text { talaciones eléctricas del hotel. }\end{array}$ \\
\hline & & $\begin{array}{l}\text { Se promueve el apagado de luces cuando } \\
\text { su uso no es estrictamente necesario. }\end{array}$ \\
\hline & & $\begin{array}{l}\text { El establecimiento está utilizando sis- } \\
\text { temas de iluminación eficientes con los } \\
\text { que se cubre al menos el } 80 \% \text { de las ne- } \\
\text { cesidades del hotel. }\end{array}$ \\
\hline & & $\begin{array}{l}\text { La empresa está utilizando algún siste- } \\
\text { ma de energía alternativa (por ejemplo } \\
\text { celdas fotovoltaicas u otros sistemas so- } \\
\text { lares) para iluminación. }\end{array}$ \\
\hline & & $\begin{array}{l}\text { Los tanques de agua caliente y las tu- } \\
\text { berías expuestas (internas cuando sea } \\
\text { posible) están recubiertos con material } \\
\text { aislante para evitar pérdidas de calor. }\end{array}$ \\
\hline & & $\begin{array}{l}\text { En la lavandería, se aprovecha el calor } \\
\text { solar para el secado de la ropa, en áreas } \\
\text { debidamente acondicionadas para este } \\
\text { propósito. }\end{array}$ \\
\hline & & $\begin{array}{l}\text { Se utilizan envases rellenables o reuti- } \\
\text { lizables para servir comidas tales como } \\
\text { recipientes para mantequilla, mermela- } \\
\text { da, miel, azúcar, salsas, otros. }\end{array}$ \\
\hline & & $\begin{array}{l}\text { En la cocina, el comedor o en el bar, se } \\
\text { utiliza menaje y accesorios de uso pro- } \\
\text { longado, evitando así productos de un } \\
\text { solo uso o desechables como vajilla de } \\
\text { Cartón, plástico o aluminio y otros. }\end{array}$ \\
\hline
\end{tabular}




\begin{tabular}{|c|c|c|}
\hline \multirow{9}{*}{ MEDIO AMBIENTE } & \multirow{9}{*}{$\begin{array}{l}\text { Administración } \\
\text { sostenible de } \\
\text { insumos de la } \\
\text { empresa }\end{array}$} & $\begin{array}{l}\text { Se utilizan productos de limpieza y pro- } \\
\text { ductos cosméticos en empaques biode- } \\
\text { gradables, reciclables o reutilizables. }\end{array}$ \\
\hline & & $\begin{array}{l}\text { Se utilizan basureros adecuados para el } \\
\text { depósito y manejo de desechos orgáni- } \\
\text { cos. }\end{array}$ \\
\hline & & $\begin{array}{l}\text { Los desechos orgánicos producidos en } \\
\text { el hotel son utilizados en un programa } \\
\text { de compostaje u otra aplicación apropia- } \\
\text { da. }\end{array}$ \\
\hline & & $\begin{array}{l}\text { La empresa dispone de recipientes ade- } \\
\text { cuados para la separación de la basura } \\
\text { (aluminio, plástico, vidrio y papel). }\end{array}$ \\
\hline & & $\begin{array}{l}\text { El personal de limpieza de habitaciones } \\
\text { separa la basura cuando el cliente no lo } \\
\text { hace. }\end{array}$ \\
\hline & & $\begin{array}{l}\text { Existe un sitio acondicionado en el cual } \\
\text { se realiza la separación final de los de- } \\
\text { sechos. }\end{array}$ \\
\hline & & $\begin{array}{l}\text { El hotel participa en un programa de } \\
\text { reciclaje al cual se envían los desechos } \\
\text { debidamente clasificados. }\end{array}$ \\
\hline & & $\begin{array}{l}\text { Los desechos sólidos generados por la } \\
\text { empresa son almacenados apropiada- } \\
\text { mente antes de su recolección final. }\end{array}$ \\
\hline & & $\begin{array}{l}\text { El establecimiento ofrece suficientes } \\
\text { zonas y habitaciones debidamente se- } \\
\text { nalizadas para fumadores y condiciones } \\
\text { para aminorar la contaminación del aire. }\end{array}$ \\
\hline \multirow[b]{2}{*}{ MEDIO AMBIENTE } & \multirow[b]{2}{*}{ Impacto del proceso } & $\begin{array}{l}\text { La reserva natural propiedad del hotel se } \\
\text { está manejando adecuadamente. }\end{array}$ \\
\hline & & $\begin{array}{l}\text { La empresa desarrolla actividades para } \\
\text { evitar la alimentación (artificial) directa } \\
\text { o indirecta a los animales silvestres. }\end{array}$ \\
\hline
\end{tabular}


Tabla 7 .

Ejes o áreas, subejes y acciones de la RSE en el Hotel Finca Campestre Rosa Blanca. Comunidad y política pública

\begin{tabular}{|l|l|l|}
\hline EJE O ÁREA & \multicolumn{1}{|c|}{ SUB EJE } & \multicolumn{1}{|c|}{ ASPECTO } \\
\hline \multirow{5}{*}{$\begin{array}{l}\text { Participación } \\
\text { en la comunidad }\end{array}$} & $\begin{array}{l}\text { La empresa contribuye y apoya la } \\
\text { formación de recursos humanos } \\
\text { para actividades complementa- } \\
\text { rias del turismo. }\end{array}$ \\
\hline $\begin{array}{l}\text { A través de material promocio- } \\
\text { nal se informa sobre actividades } \\
\text { benéficas que se desarrollan en la } \\
\text { comunidad. }\end{array}$ \\
$\begin{array}{l}\text { Ca empresa facilita transporte te- } \\
\text { rrestre o marítimo a personas de } \\
\text { la comunidad con frecuencia o en } \\
\text { casos de emergencia. }\end{array}$ \\
\hline $\begin{array}{l}\text { Los rótulos dedicados a la orien- } \\
\text { tación y promoción de la empresa } \\
\text { no interfieren con el medio cultu- } \\
\text { ral, social o ambiental. }\end{array}$ \\
\hline & $\begin{array}{l}\text { La empresa utiliza personas de la } \\
\text { localidad para cubrir más del 60\% } \\
\text { de las necesidades de personal. }\end{array}$ \\
\hline $\begin{array}{l}\text { La empresa aprovecha y promue- } \\
\text { ve el uso y consumo de insumos } \\
\text { producidos a nivel local. }\end{array}$ \\
\hline $\begin{array}{l}\text { Para adornos del hotel y de las ha- } \\
\text { bitaciones se utilizan artesanías u } \\
\text { otro tipo de manifestaciones ar- } \\
\text { tísticas producidas local, regional } \\
\text { o nacionalmente. }\end{array}$ \\
$\begin{array}{l}\text { La empresa ha realizado o realiza } \\
\text { contribuciones económicas o en } \\
\text { especie a la comunidad para obras } \\
\text { específicas de infraestructura o } \\
\text { su mantenimiento. }\end{array}$ \\
\hline
\end{tabular}




\begin{tabular}{|c|c|c|}
\hline \multirow{4}{*}{ COMUNIDAD } & Trabajo voluntario & $\begin{array}{l}\text { Se trabaja con estudiantes que } \\
\text { vienen propiamente de Estados } \\
\text { Unidos a realizar sus trabajos } \\
\text { comunales universitarios con la } \\
\text { comunidad en actividades pro- } \\
\text { movidas por el hotel. Un ejemplo } \\
\text { es la enseñanza de inglés para } \\
\text { miembros de la comunidad y la } \\
\text { escuela. }\end{array}$ \\
\hline & Liderazgo social & $\begin{array}{l}\text { El hotel ejecuta acciones prácticas } \\
\text { para garantizar la seguridad de } \\
\text { los turistas y empleados. }\end{array}$ \\
\hline & \multirow{2}{*}{ Patrimonio socio cultural } & $\begin{array}{l}\text { La empresa participa activamente } \\
\text { contribuyendo con el desarrollo } \\
\text { de actividades deportivas, artísti- } \\
\text { cas, culturales. }\end{array}$ \\
\hline & & $\begin{array}{l}\text { En la promoción de la empresa se } \\
\text { integran los elementos culturales } \\
\text { de la región y de las comunidades } \\
\text { locales. }\end{array}$ \\
\hline \multirow{2}{*}{$\begin{array}{l}\text { POLÍTICA } \\
\text { PÚBLICA }\end{array}$} & Transparencia política & $\begin{array}{l}\text { Estar al día con el pago de los } \\
\text { impuestos (renta, bienes e in- } \\
\text { muebles, sociedades anónimas y } \\
\text { otros). }\end{array}$ \\
\hline & $\begin{array}{l}\text { Proyectos conjuntos } \\
\text { (alianza pública y privada) }\end{array}$ & $\begin{array}{l}\text { La empresa se involucra para in- } \\
\text { tegrar asociaciones o comités que } \\
\text { trabajen en pro de mejoras para la } \\
\text { localidad donde opera. }\end{array}$ \\
\hline
\end{tabular}

Nota: Instrumentos aplicados (2014).

Como se puede observar en las tablas anteriores, el hotel a nivel de RSE está a derecho con la normativa jurídica del país. Cumple con los códigos del sector turismo como son no al trabajo infantil, no a la explotación sexual de menores de edad, tiene iniciativas comunales, tal como la participación en ferias en pro de la comunidad.

El apoyo contante a la protección del ambiente, el fortalecimiento a la comunidad y el aprovechamiento de los recursos de la región benefician a los vecinos como proveedores, trabajadores directos, empresas de apoyo, organizaciones con alianzas estratégicas como con la escuela y el colegio de Santa Bárbara. Un 
ejemplo muy loable es la alianza que se mencionó anteriormente con el hogar de ancianos de la comunidad, donde los adultos mayores hacen artesanías y esas se ponen a la venta en la tienda del hotel (alfombras). Esos recursos son trasladados al hogar para cubrir algunas necesidades.

Al cumplir con los ejes, áreas y aspectos de la RSE, se logra apreciar la articulación entre los principios y el hotel, en la siguiente tabla se presenta este enlace:

Tabla 8 .

Principios de la RSE en el Hotel Finca Campestre Rosa Blanca

\begin{tabular}{|c|c|}
\hline PRINCIPIOS & ASPECTOS \\
\hline $\begin{array}{l}\text { RENDICIÓN } \\
\text { DE CUENTAS }\end{array}$ & $\begin{array}{l}\text { Asamblea de socios. } \\
\text { Presentación de estados financieros ante el Ministerio de } \\
\text { Hacienda, Instituto Nacional de Seguros, Caja Costarri- } \\
\text { cense de Seguro Social, Instituto Mixto de Ayuda Social, } \\
\text { Instituto Nacional de Aprendizaje, Banco Popular y Desa- } \\
\text { rrollo Comunal. } \\
\text { Actualización de datos ante las entidades financieras del } \\
\text { país. } \\
\text { Legitimación de capital. }\end{array}$ \\
\hline TRANSPARENCIA & $\begin{array}{l}\text { Para poder obtener la CST, el hotel debe mostrar la docu- } \\
\text { mentación administrativa, financiera contable, tributaria } \\
\text { y ambiental. } \\
\text { Para ser proveedor del Estado también debe cumplir con } \\
\text { lo establecido en el Gobierno digital en cuanto a Merlink } \\
\text { o Compr@Red. }\end{array}$ \\
\hline
\end{tabular}




\begin{tabular}{|c|c|}
\hline \multirow{13}{*}{$\begin{array}{l}\text { COMPORTAMIENTO } \\
\text { ÉTICO }\end{array}$} & Constitución Política de la República de Costa Rica. \\
\hline & Aplicación del Código de Trabajo (cliente interno). \\
\hline & Aplicación del Código de Conducta (cliente externo). \\
\hline & Aplicación del Código Sísmico (medio ambiente). \\
\hline & $\begin{array}{l}\text { Aplicación del Código de la Niñez y de la Adolescencia } \\
\text { (comunidad). }\end{array}$ \\
\hline & Aplicación de la Ley del Ambiente (medio ambiente). \\
\hline & $\begin{array}{l}\text { Aplicación de la Ley de Igualdad de oportunidades para } \\
\text { las personas con discapacidad (cliente interno y externo, } \\
\text { comunidad). }\end{array}$ \\
\hline & Ley reguladora del mercado de valores (transparencia). \\
\hline & Código de comercio (rendición de cuentas, proveedores). \\
\hline & $\begin{array}{l}\text { Ley de Promoción de la Competencia y Defensa Efectiva } \\
\text { Consumidor (transparencia, rendición de cuentas, provee- } \\
\text { dores). }\end{array}$ \\
\hline & Sistema Nacional para la Calidad (clientes, proveedores). \\
\hline & $\begin{array}{l}\text { Ley de Promoción de la Competencia y Defensa Efectiva } \\
\text { del Consumidor (proveedores, comunidad). }\end{array}$ \\
\hline & $\begin{array}{l}\text { Reglamento para otorgar el Certificado para la Sostenibi- } \\
\text { lidad Turística (CST). }\end{array}$ \\
\hline $\begin{array}{l}\text { RESPETO } \\
\text { POR LOS INTERÉSES }\end{array}$ & $\begin{array}{l}\text { Accionistas, proveedores, clientes, comunidad, municipa- } \\
\text { lidad, gobierno central, instituciones autónomas, asocia- } \\
\text { ciones, fundaciones, competencia. }\end{array}$ \\
\hline $\begin{array}{l}\text { RESPETO POR EL } \\
\text { PRINCIPIO DE } \\
\text { LEGALIDAD }\end{array}$ & $\begin{array}{l}\text { Cumplimiento con el bloque legal del país, del sector y } \\
\text { de la comunidad en cuanto a la parte laboral, ambiental, } \\
\text { turística, comercial, tecnológica, financiera. }\end{array}$ \\
\hline $\begin{array}{l}\text { RESPETO A LA } \\
\text { NORMATIVA } \\
\text { INTERNACIONAL DE } \\
\text { COMPORTAMIENTO }\end{array}$ & $\begin{array}{l}\text { Organización Internacional del Trabajo, Organización } \\
\text { Mundial de Turismo, Secretaria Internacional de Ética en } \\
\text { Turismo, Organización Panamericana para la Salud, So- } \\
\text { ciedad Internacional de Ecoturismo. }\end{array}$ \\
\hline
\end{tabular}

Nota: Instrumentos aplicados (2015). 
Como se puede apreciar, la forma en que se articulan los principios de la RSE son simples y demuestran que al estar acreditada con CST, a su vez, es un hotel con RSE.

\section{Conclusiones}

El Hotel Finca Campestre Rosa Blanca, al contar con el nivel máximo de CST y a la vez con una puntuación superior, en forma implícita hace RSE, porque la Certificación de Sostenibilidad Turística ha sido visionaria con respecto al turismo responsable, también faculta para que el Hotel pueda hacer RSE en la comunidad, con el medio ambiente, con representantes del Estado y, ante todo, con los clientes internos y clientes externos.

En este caso y de acuerdo con los diferentes datos obtenidos con entrevistas y otros instrumentos para recolectar información, el hotel ha tenido un buen desenvolvimiento en los diferentes ámbitos que involucran la CST y la RSE, y esto se debe a que ambas se complementan y se fortalecen entre sí.

Las estrategias que se implementan favorecen a todas las partes involucradas y eso permite que la RSE de la empresa hotelera sea visible y reconocible.

Lo anterior significa que una empresa que está empoderada con la sostenibilidad, también está empoderada con la responsabilidad social empresarial, porque ambas estrategias se desarrollan para que el entorno natural, las personas (trabajadores, visitantes, vecinos, proveedores, y otros), la construcción o edificio y la gestión misma de la organización se integren entre sí.

Dicho de otra manera, ambas estrategias tienen elementos en común, como son: El medio ambiente, las estrategias de gestión organizativa en pro de la generación de bienes y servicios turísticos, el manejo de proveedores, el uso de los recursos de la zona o región, el aprovechamiento responsable de los recursos en todos los sentidos: humanos, financieros, económicos, tecnológicos ambientales, la participación con la comunidad, la calidad de vida de los trabajadores, el respeto por la normativa nacional e internacional.

La recomendación más importante es realizar otra investigación hacia las escalas de medición y así ver su potencial homologación. 


\section{Referencias}

Asociación Empresarial de Desarrollo (AED). (2004). Responsabilidad social empresarial definición de términos. Cámara de Industria de Costa Rica. San José, Costa Rica.

Asociación Empresarial de Desarrollo (AED). (2013). Jornada Anual de Sostenibilidad Costa Rica camino a la OCDE. Cámara Nacional de Turismo de Costa Rica. San José, Costa Rica.

ALIARSE. (2009). Alianza para el Desarrollo: Motor de la responsabilidad social empresarial casos de organizaciones públicas y privadas Costa Rica. San José, Costa Rica: Fundación para la Sostenibilidad y Equidad ALIARSE.

Barquero, M. (Enero, 2016). Costa Rica recibió 2.665.000 turistas en el 2015. Periódico La Nación. San José, Costa Rica.

Bien, A. (2008). Una guía básica sobre la acreditación de programas de Certificación de programas de turismo sostenible. Rainforest Alliance. MIF Fomin.

Comunidad de Estados Latinoamericanos y Caribeños. (2015). Memoria de la III Cumbre CELAC. San José Costa Rica.

Domínguez, J. (2012). Conceptualización sobre la responsabilidad social específica de una universidad católica. En J. Domínguez, C. Rama (Eds.), La responsabilidad social universitaria en la educación a distancia, (pp. 53-74). Chimbote, Perú: ULADECH Católica.

Flores, A. (2014). Compendio de los logros más importantes del Ministro de Turismo en el período 2011-2014 (Informe Final de Gestión). San José, Costa Rica.

Grant, R. (2010). Contemporary strategy analysis. Text and cases (7th Ed.). Published by John Wiley \& Son Ltd. United Kingdown.

Instituto Costarricense de Turismo. (2015). Estadísticas de entradas y saldas de turistas (Informe parcial). ICT. San José, Costa Rica.

Instituto Costarricense de Turismo. (2014). Informe de avance sobre el Plan Nacional de Turismo 2010-2016. ICT. San José, Costa Rica.

Instituto Costarricense de Turismo. (2012). Caracteristicas generales de los turistas que visitan Costa Rica. Macroproceso de Planeamiento y Desarrollo Turístico del ICT.

Instituto Costarricense de Turismo. (2010). Plan Nacional de Turismo 2010- 2016.

ICT. San José, Costa Rica. 
Lindberg, K. and Aylward, B. (1999). Price Responsiveness in the developing country nature tourism context: Review and Costa Rican. case study. Journal of Leisure Research, 31(3), 281-300.

Napier, D. (1997). Green scene. The Ecological paradise of Costa Rica is taking its first steps towards serving coporate and incentive markets. Meetings and Incentive Travel, 26(3), 45-58.

Reficco, E. y Ogliastri E. (2009). Empresa y sociedad en América Latina (Introducción). Revista Latinoamericana de Administración, Segundo Semestre 43, 1-198. Recuperado de http://revistaadcademica.cladea org

Porter, M., y Kramer, M. (2006). Strategy and Society: The link between competitive advantage and Corporate Social Responsibility. Harvard Business Review, 84, 78-92.

Riotte, I. (2015). Definición de empresas denominadas "DMC" (empresas organizadoras de eventos empresariales con responsabilidad social). San José, Costa Rica.

Singh P.J, Power D. and Chuong, S.C. (2011). A resource dependence theory perspective of ISO 9000 in managing organizational environment. Journal of Operations Management, 29(1-2), 49-64. 